Check for updates

Cite this: RSC Adv., 2019, 9, 30389

\section{LncRNA ZEB1-AS1 inhibits renal fibrosis in diabetic nephropathy by regulating the miR-217/MAFB axis $\uparrow$}

\begin{abstract}
Yan Song, Chunxia Miao and Jianwen Wang (D) *
Diabetic nephropathy (DN) is a common chronic microvascular complication of diabetes, characterized by the deposition of extracellular matrix (ECM) proteins. Zinc finger E-box binding homeobox 1 antisense 1 (ZEB1-AS1) has been implicated in kidney fibrosis of human DN. Here, we further explored the detailed molecular mechanism of ZEB1-AS1 in renal fibrosis in DN. The expression of ZEB1-AS1 was monitored using a quantitative real-time polymerase chain reaction. Blood glucose concentrations of mice were measured using a fast blood glucose meter. The high-performance liquid chromatography method was used to measure the serum creatinine level. Western blot analysis was used to detect the protein level of $\alpha$-smooth muscle actin ( $\alpha$-SMA), fibronectin, collagen I, collagen IV and MAFB. The level of urine albumin was detected using the BCG (Bromocresol Green) albumin assay kit. The interaction between ZEB1-AS1, miR-217 and MAFB was investigated via the luciferase reporter and RNA immunoprecipitation analysis. Decreased ZEB1-AS1 expression in DN patients and db/db diabetic mice as well as in high glucose (HG)induced HK-2 cells was detected. Reduction in the $\alpha$-SMA, fibronectin, collagen I and IV protein expression, induced by the overexpressed ZEB1-AS1, was found in db/db diabetic mice and HG-induced HK-2 cells. In addition, we discovered that ZEB1-AS1 directly targeted miR-217, and MAFB was the target of miR-217; thus, ZEB1-AS1 might regulate the MAFB expression by targeting miR-217. Furthermore, functional experiments indicated that overexpressed ZEB1-AS1 might have decreased the accumulation of ECM proteins in the HG-induced HK-2 cells by regulating the miR-217 and MAFB expression. Overexpressed ZEB1-AS1 may inhibit renal fibrosis in diabetic nephropathy by regulating the miR-217/ MAFB axis, identifying novel therapeutic targets for renal fibrosis in diabetic nephropathy.
\end{abstract}

Received 20th July 2019 Accepted 20th August 2019 DOI: 10.1039/c9ra05602e rsc.li/rsc-advances which are involved in the transcription, pre-mRNA processing, and translation of genes. ${ }^{5}$ To date, accumulating evidence suggests the importance of the regulatory function of lncRNAs in the pathological processes of human diseases, including those of DN. ${ }^{6,7}$ Zinc finger E-box binding homeobox 1 antisense 1 (ZEB1-AS1) is a cancer-related IncRNA, which functions as an oncogenic regulator in diverse malignancies. The dysregulation of ZEB1-AS1 has been exhibited to contribute to the oncogenesis and disease progression, indicating the potential clinical value of ZEB1-AS1 as a promising biomarker or target for tumor therapy ${ }^{8,9}$ Recently, it was shown that p53, ZEB1-AS1 and ZEB1 signaling may be involved in renal fibrosis in human DN. ${ }^{10}$ However, the role and the molecular mechanism of ZEB1-AS1 in kidney fibrosis of human DN are still not fully understood.

MicroRNAs (miRNAs) are small noncoding RNA molecules with the length of 20-22 nucleotides. They can participate in the post-transcriptional control of the target mRNA by predominantly binding to the $3^{\prime}$ untranslated regions (UTR). ${ }^{11}$ Many miRNAs have been shown to be involved in the pathogenesis of DN. ${ }^{12}$ Recently, it was revealed that miR-217 is implicated in the development of the diabetic kidney disease by promoting chronic inflammation, renal fibrosis, and angiogenesis. ${ }^{13}$ The repression of miR-217 protected against high glucose-induced
Department of Nephrology, People's Hospital of Rizhao, No. 126, Taian Road, Rizhao, Shandong, 276800, China. E-mail: yilonghanjnpvy@163.com; Tel: +860633 3365610 $\uparrow$ Electronic supplementary information (ESI) available. See DOI: 10.1039/c9ra05602e 
podocyte and glomerular mesangial cell fibrosis by regulating the underlying pathway, ${ }^{\mathbf{1 4 , 1 5}}$ while the role of miR-217 in tubular cell fibrosis remains unclear. MAFB, containing a basic leucine zipper, is a member of the large Maf family and can mediate dimer formation and target DNA binding to the Maf recognition element (MARE). ${ }^{\mathbf{1 6}}$ Previous studies have shown that MAFB plays an important role in the podocyte differentiation and its foot process formation, ${ }^{17}$ and mutations of MAFB inhibit the maintenance and development of podocytes, which could lead to carpotarsal osteolysis (MCTO) and focal segmental glomerulosclerosis (FSGS). ${ }^{\mathbf{1 8 , 1 9}}$ Additionally, recent studies showed that overexpressed MAFB in podocytes prevented the development of $\mathrm{DN},{ }^{20}$ and miR-320a could aggravate renal dysfunction in $\mathrm{DN}$ by targeting MAFB. ${ }^{21}$

In the present study, $\mathrm{db} / \mathrm{db}$ diabetic mice and high glucose (HG)-induced HK-2 cells were used to investigate the biological function and the potential molecular regulation mechanism of ZEB1-AS1 in the renal fibrosis progression of DN patients, which might indicate novel therapeutic targets for renal fibrosis in $\mathrm{DN}$.

\section{Materials and methods}

\subsection{Collection of human renal tissues}

The DN renal tissues were obtained from $26 \mathrm{DN}$ patients at the Department of Nephrology, People's Hospital of Rizhao. DN was diagnosed based on the presence of diabetes, massive proteinuria, and other histological changes typical of $\mathrm{DN}^{22}$ The normal tissues around the tumor from 10 patients with kidney carcinoma without diabetes were collected to be used as the normal control. This study was approved by the Ethics Committee of the Department of Nephrology, People's Hospital of Rizhao. Written informed consent was collected from all patients and the hospital.

\subsection{Animals}

The $\mathrm{db} / \mathrm{db}$ mouse (diabetic mouse) with genetic defects in the leptin receptor was derived from the autosomal recessive inheritance of the $\mathrm{C} 57 \mathrm{BL} / \mathrm{Ks}$ inbred mating and belongs to the type II diabetes model, characterized by excessive obesity, hyperglycemia, insulin resistance and lipid metabolism disorders. Furthermore, based on the typical renal pathological changes, as well as elevated serum creatinine, urinary albuminuria and proteinuria, $\mathrm{db} / \mathrm{db}$ mice are widely used as the DN model mice. In the present study, $\mathrm{db} / \mathrm{db}$ mice and their littermate $\mathrm{db} / \mathrm{m}$ mice (male; 4 week old) were obtained from NBRI (Nanjing, China). The mice were divided into four groups $(n=6$ for each group): $\mathrm{db} / \mathrm{m}$ group (control), $\mathrm{db} / \mathrm{db}$ group (DN group), $\mathrm{db} / \mathrm{db}+$ pcDNA group (DN treated with pcDNA) and $\mathrm{db} / \mathrm{db}+$ ZEB1-AS1 group (DN treated with ZEB1-AS1). When the mice reached eight weeks of age, the $\mathrm{db} / \mathrm{db}+$ pcDNA group and the $\mathrm{db} / \mathrm{db}+\mathrm{ZEB} 1-A S 1$ group were injected with either the pcDNA or ZEB1-AS1 plasmid (Genepharma, Shanghai, China) via the tail vein at a dose of $15 \mathrm{mgkg}^{-1}$ day every second day for 4 weeks until the urinary microalbumin level of the $\mathrm{db} / \mathrm{db}+\mathrm{ZEB} 1-\mathrm{AS} 1$ group was lower than that of the untreated $\mathrm{db} / \mathrm{db}$ group.
Finally, the renal tissues of mice in each group were isolated for further study. This study was approved by the Ethics Committee of Animal Research Institute of Department of Nephrology, People's Hospital of Rizhao and performed in accordance with the guidelines of the National Institutes of Health Guide for the Care and Use of Laboratory Animals.

\subsection{Cell culture and high glucose treatment}

HK-2 cell line was purchased from the Chinese Academy of Sciences Shanghai Cell Bank (Shanghai, China) and cultured in DMEM/F12 (Gibco, Carlsbad, CA, USA) supplemented with 10\% fetal bovine serum (Gibco) at $37{ }^{\circ} \mathrm{C}$ with $5 \% \mathrm{CO}_{2}$. When the cells were grown to $70 \%$ confluency, high glucose (HG; $33.3 \mathrm{mM}$ glucose) or normal glucose ( $\mathrm{NG} ; 5.5 \mathrm{mM}$ glucose) was utilized to treat the cells for different time periods.

\subsection{Cell transfection}

Small interfering RNA (siRNA) targeting ZEB1-AS1 (si-ZEB1AS1), siRNA targeting MAFB (si-MAFB), siRNA negative control (si-NC), pcDNA and pcDNA- ZEB1-AS1 overexpression vector (ZEB1-AS1) were synthesized by Genepharma (Shanghai, China). The miR-217 mimic or the inhibitor (miR-217 or antimiR-217) and the corresponding negative control (miR-NC or anti-miR-NC) were obtained from RIBOBIO (Guangzhou, China). These oligonucleotides or vectors were transfected into HK-2 cells after the treatment with HG or NG using Lipofectamine 2000 Transfection Reagent (Invitrogen, Carlsbad, CA, USA). Cell transfection was repeated three times.

\subsection{Quantitative real-time polymerase chain reaction (qRT- PCR)}

Total RNA was extracted from tissues and cells using Trizol Reagent (Invitrogen) according to the manufacturer's instructions. For ZEB1AS1 expression detection, cDNA was synthesized using M-MLV Reverse Transcriptase (Invitrogen). For miR-217 level detection, total RNA was reverse-transcribed using One Step PrimeScript ${ }^{\circledR}$ miRNA cDNA Synthesis Kit (Takara, Dalian, China). Then real-time qPCR was performed by using SYBR Green Realtime PCR Master Mix (Takara). The fold change was analyzed using the $2^{-\Delta \Delta C_{t}}$ method and normalized by GAPDH or U6. All experiments were performed three times, and the average value was taken. The special primers for miR-217 or U6 were purchased from GeneCopoeia, and the primers for ZEB1-AS1 or GAPDH were labeled as follows: ZEB1AS1, 5'-CCGTGGGCACTGCTGAAT-3' (forward) and 5'-CTGCTGGCAAGCGGAACT-3' (reverse); GAPDH, $5^{\prime}$-GGTCTCCTCTGACTTCAACA- ${ }^{\prime}$ (forward) and $5^{\prime}$-GTGAGGGTCTCTCTCTTCCT-3' (reverse).

\subsection{Analysis of renal biochemical indicators}

The blood glucose concentration of mice was examined using a fast blood glucose meter. The level of urine albumin was detected using the BCG (Bromocresol Green) albumin assay kit. The high-performance liquid chromatography (HPLC) method was used to measure the serum creatinine level. The ACR level 
was evaluated using the method described previously. ${ }^{23}$ Experiments were repeated three times to evaluate the mean values.

\subsection{Western blot assay}

Total proteins were extracted from cells and tissues using the RIPA Lysis Buffer (Beyotime, Shanghai, China) according to the manufacturer's protocol and quantified using a bicinchoninic acid (BCA) Protein Assay Kit (Beyotime). Total proteins were separated by $10 \%$ sodium dodecyl sulphate-polyacrylamide gel electrophoresis (SDS-PAGE), transferred to a polyvinylidene difluoride membrane (PVDF; Merck Millipore, Billerica, MA, USA) and then blocked with $5 \%$ skimmed milk for $1 \mathrm{~h}$ at room temperature. Subsequently, the membrane was incubated with primary antibodies against $\alpha$-smooth muscle actin ( $\alpha$-SMA) (1:1000, Abcam, ab32575), fibronectin (1:500, Abcam, ab2413), collagen I (1:1000, Abcam, ab34710), collagen IV (1:1000, Abcam, ab6586), MAFB $(1: 1000$, Cell Signaling Technology, 79737S) or $\beta$-actin $\left(1: 1000\right.$, Abcam, ab5694) at $4{ }^{\circ} \mathrm{C}$ overnight and incubated with the horseradish peroxidaseconjugated secondary antibody $(1: 2000$, Abcam, ab6728) for $2 \mathrm{~h}$ at room temperature after being washed. Finally, the blots were visualized using the ECL Western Blotting Substrate (Beyotime). The experiments were performed three times independently.

\subsection{Luciferase reporter assay}

The ZEB1-AS1 mRNA and MAFB 3'-UTR containing wild-type (WT) or mutant (MUT) binding sequence of miR-217 were cloned into the pmirGLO basic vectors (Promega, Shanghai, China). Then, the HK-2 cells were seeded in 24-well plates for $24 \mathrm{~h}$ and co-transfected with ZEB1-AS1-WT or ZEB1-AS1-MUT or MAFB-WT or MAFB-MUT with miR-217 mimics or miR-NC, respectively, using Lipofectamine 2000. Finally, a Dual Luciferase Assay Kit (Promega) was utilized to evaluate the luciferase activity following the manufacturer's instructions.

\subsection{RNA immunoprecipitation assay}

The RNA immunoprecipitation (RIP) assay was performed to verify the binding of ZEB1-AS1, miR-217 and MAFB. The HK-2 cells, transfected with miR-217 or miR-NC, were lysed in the RIP buffer, and the cell lysis solution was incubated with magnetic beads conjugated with human anti-Ago2 (Millipore) or the negative control mouse IgG antibody (CST, USA). Subsequently, the enrichment of ZEB1-AS1 or MAFB was quantified by qRT-PCR.

\subsection{Wound healing assays}

A wound healing assay was used to analyze cell migration. Cells were cultured on 24-well plates and wounded using a pipette in a cell monolayer. Wounded areas were photographed at $0 \mathrm{~h}$ and $48 \mathrm{~h}$, and the migratory distance of the cells was recorded to quantify their migration rate.

\subsection{Statistical analysis}

Statistical analysis was performed using GraphPad Prism 7 (GraphPad Inc., San Diego, CA, USA). The unpaired Student's $t$ - test and one-way analysis of variance were used to analyze the difference between the groups. The data of at least three independent experiments are presented as the mean \pm standard error of the mean (SEM). $P<0.05$ was considered statistically significant.

\section{Results}

3.1. Decreased ZEB1-AS1 expression in DN patients and db/ db diabetic mice

ZEB1-AS1 expression in DN patients and $\mathrm{db} / \mathrm{db}$ mice renal tissues was detected, and a significantly reduced ZEB1-AS1 expression compared to that in normal renal tissues was found (Fig. 1A and B).

\subsection{Overexpressed ZEB1-AS1 mitigates renal dysfunction and fibrosis in $\mathrm{db} / \mathrm{db}$ diabetic mice}

In order to explore the effect of ZEB1-AS1 on DN fibrosis, $\mathrm{db} / \mathrm{db}$ model mice were obtained by injection via the tail vein with either ZEB1-AS1 plasmid or pcDNA. Highly expressed ZEB1-AS1 was found in the serum of the $\mathrm{db} / \mathrm{db}+$ ZEB1-AS1 and $\mathrm{db} / \mathrm{m}$ groups (Fig. 2A). Subsequently, we found that the $\mathrm{db} / \mathrm{db}$ diabetic mice displayed higher levels of blood glucose, serum creatinine, and urinary albumin-to-creatinine ratio (ACR), which was obviously reversed by the ZEB1-AS1 treatment (Fig. 2B-D). Furthermore, the protein expression of $\alpha$-SMA, fibronectin, collagen I and IV was detected, and the results showed a markedly increased expression in the $\mathrm{db} / \mathrm{db}$ diabetic mice, which was also attenuated by the ZEB1-AS1 treatment (Fig. 2E and F). These results indicated that ZEB1-AS1 could mitigate renal dysfunction and fibrosis in $\mathrm{db} / \mathrm{db}$ diabetic mice.

\subsection{Overexpressed ZEB1-AS1 attenuates HG-induced fibrosis in HK-2 cells}

To elucidate the role of ZEB1-AS1 in HG-induced renal fibrosis, ZEB1-AS1 or pcDNA was transfected into the HK-2 cells after the treatment with HG or NG. Subsequently, we discovered that the HG treatment significantly reduced the expression of ZEB1-AS1 but enhanced the accumulation of $\alpha$-SMA, fibronectin, collagen I and IV, which was significantly attenuated by the ZEB1-AS1 treatment (Fig. 3A-C). Additionally, a wound healing assay was performed to detect cell migration, and the results showed that the overexpressed ZEB1-AS1 partially reversed the HGinduced cell migration (Fig. S1†), indicating the change of phenotypic consequence to alter the ZEB1-AS1 levels. Thus, these findings suggested that the overexpressed ZEB1-AS1 might protect against HG-induced renal fibrosis in HK-2 cells, which was consistent with the in vivo results.

\subsection{ZEB1-AS1 directly targets miR-217 and suppresses miR- 217 expression}

To investigate the underlying molecular mechanism involved in the ZEB1-AS1-mediated protective effect of HG-induced fibrosis in HK-2 cells, a bioinformatics analysis was performed and miR217 was predicted to be the target of ZEB1-AS1 with putative binding sites (Fig. 4A). To prove this prediction, luciferase 
A

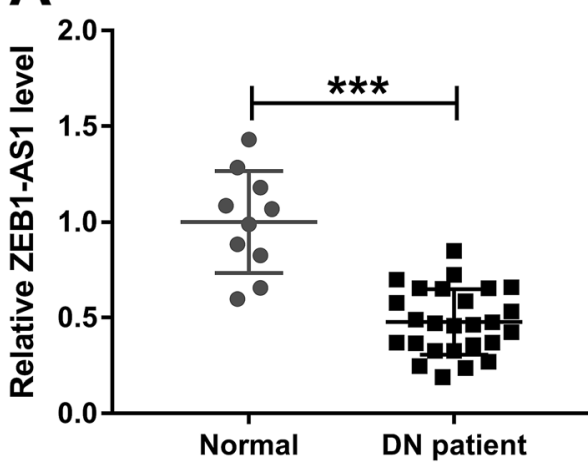

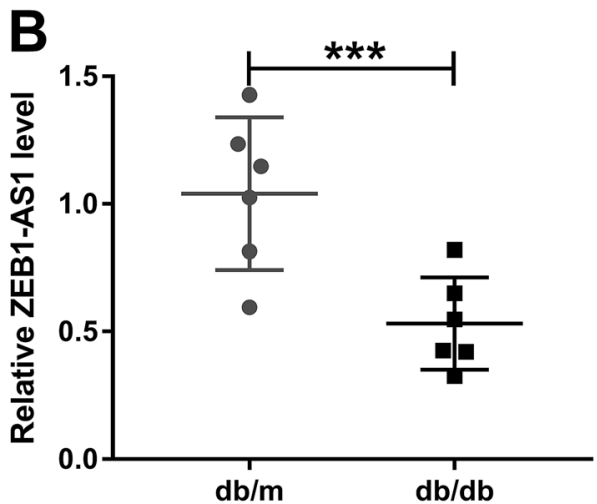

$\mathrm{db} / \mathrm{m}$

$\mathrm{db} / \mathrm{db}$

Fig. 1 The expression of ZEB1-AS1 in DN patients and db/db diabetic mice. (A and B) ZEB1-AS1 expression in DN and normal renal tissues (A), as well as in $\mathrm{db} / \mathrm{db}$ and $\mathrm{db} / \mathrm{m}$ mice (B) was detected using qRT-PCR. $* P<0.05$; $* * P<0.01 ; * * * P<0.001$.

reporter analysis and RIP assay were carried out, and the results indicated that the miR-217 mimic reduced the luciferase activity of the ZEB1-AS1-WT reporter vector but not the activity of the ZEB1-AS1-MUT reporter vector in HK-2 cells (Fig. 4B). In the meanwhile, the overexpression of miR-217 led to obvious enrichment of ZEB1-AS1 after Ago2 RIP but not after IgG RIP (Fig. 4C). Furthermore, we found that the expression of miR-217 was significantly inhibited by ZEB1-AS1, but enhanced by siZEB1-AS1 in HK-2 cells (Fig. 4D). To sum up, we found that ZEB1-AS1 deliberately suppressed the miR-217 expression.
3.5. Overexpressed ZEB1-AS1 protects against HG-induced renal fibrosis in HK-2 cells by regulating miR-217

MiR-217 being a target of ZEB1-AS1, we hypothesized that ZEB1AS1 might exert its protective effect in HG-induced renal fibrosis via regulating the miR-217 expression. To validate this hypothesis, HK-2 cells were treated with HG or NG and transfected with pcDNA, ZEB1-AS1, ZEB1-AS1 and miR-NC or ZEB1AS1 and miR-217, respectively. Interestingly, we found that the expression of miR-217 was significantly increased by the HG treatment but inhibited by the transfection of ZEB1-AS1 (Fig. 5A

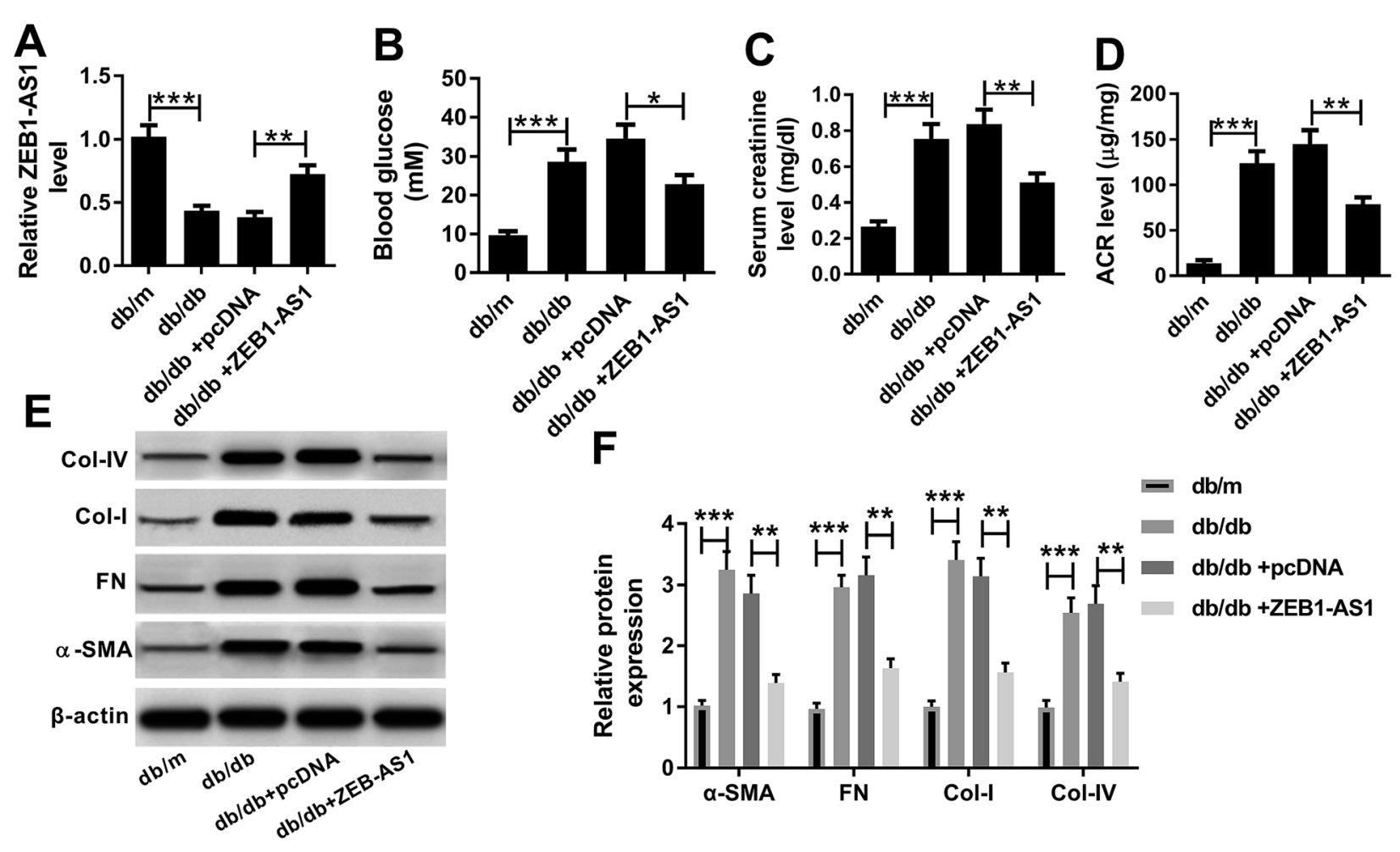

Fig. 2 Overexpressed ZEB1-AS1 mitigates renal dysfunction and fibrosis in db/db diabetic mice. (A) The expression of ZEB1-AS1 was measured in $\mathrm{db} / \mathrm{m}$ group, $\mathrm{db} / \mathrm{db}$ group, $\mathrm{db} / \mathrm{db}+\mathrm{pcDNA}$ group and db/db + ZEB1-AS1 group using qRT-PCR. (B and C) The blood glucose and serum creatinine concentration in mice was examined using a fast blood glucose meter or HPLC method in each group, respectively. (D) The ACR level of mice was calculated. (E) Western blot analysis was used to detect the level of $\alpha$-SMA, fibronectin, collagen I and collagen IV in each group. (F) Densitometric quantification of the Western blot bands. ${ }^{*} P<0.05 ; * * P<0.01 ; * * * P<0.001$. 

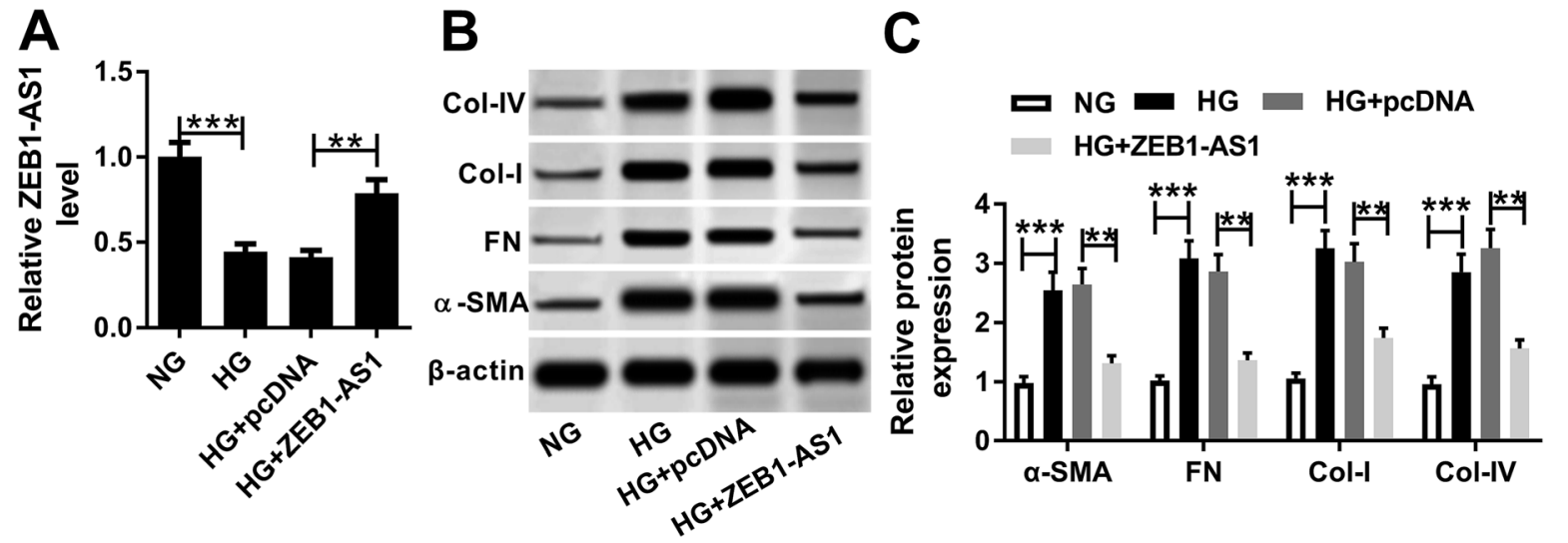

Fig. 3 Overexpressed ZEB1-AS1 attenuates HG-induced fibrosis in HK-2 cells. ZEB1-AS1 or pcDNA was transfected into HK-2 cells after treatment with HG or NG. (A) The expression of ZEB1-AS1 was detected. (B and C) The levels of $\alpha$-SMA, fibronectin, collagen I and collagen IV were evaluated using the Western blot analysis. $* P<0.05 ; * * P<0.01 ; * * P<0.001$.

and B). Then, we also found that the ZEB1-AS1-mediated inhibition of the $\alpha$-SMA, fibronectin, collagen I and IV protein expression was significantly attenuated by the miR-217 transfection (Fig. 5C and D). Therefore, we confirmed that the overexpressed ZEB1-AS1 might protect against HG-induced renal fibrosis by targeting the miR-217 inhibition in HK-2 cells.

3.6. MiR-217 directly targets MAFB and negatively regulates its expression

According to the prediction of the DIANA TOOL database, MAFB was identified as a potential target of miR-217 with the putative binding sites (Fig. 6A). Then, the luciferase reporter analysis showed that the miR-217 mimic reduced the luciferase activity of the MAFB-WT reporter vector but not the activity of the MAFBMUT reporter vector in HK-2 cells (Fig. 6B). Subsequently, the RIP assay data indicated that the miR-217 mimic led to an obvious enrichment of MAFB after Ago2 RIP, while there was no change after IgG RIP (Fig. 6C). Additionally, the miR-217 mimic depressed the MAFB protein expression while the miR-217 inhibitor promoted the MAFB expression in HK-2 cells (Fig. 6D). Therefore, miR-217 suppressed the MAFB expression in a targeted manner.

3.7. Overexpressed ZEB1-AS1 protects against HG-induced renal fibrosis in HK-2 cells by regulating miR-217/MAFB axis

The expression of MAFB was detected in HK-2 cells after the treatment with $\mathrm{HG}$ or $\mathrm{NG}$, and we found that the HG treatment
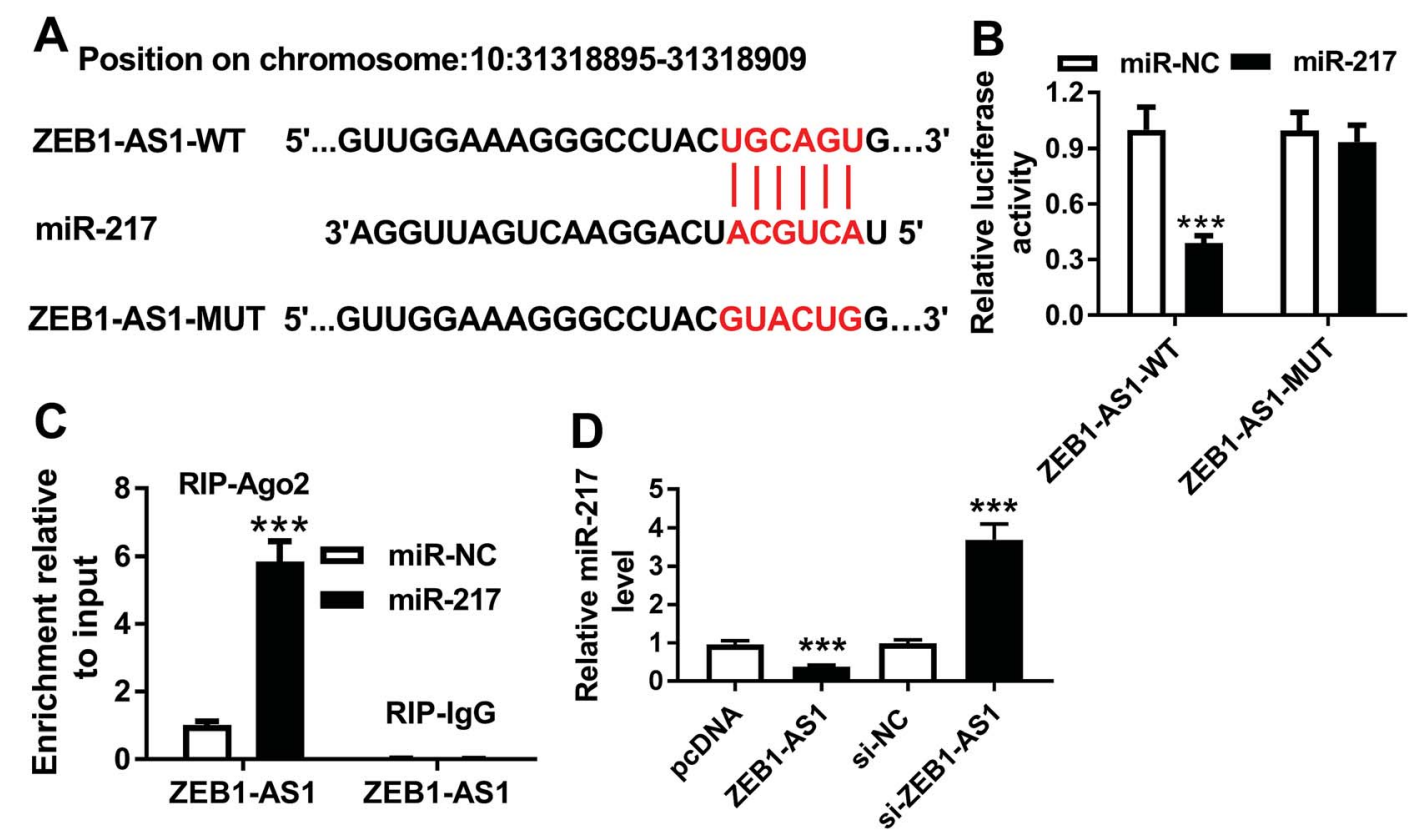

Fig. 4 ZEB1-AS1 directly targets miR-217 and suppresses its expression. (A) The putative binding sites of ZEB1-AS1 and miR-217 are listed. (B) Luciferase activity was detected in HK-2 cells co-transfected with ZEB1-AS1-WT or ZEB1-AS1-MUT and miR-217 or miR-NC. (C) The enrichment of ZEB1-AS1 was detected in HK-2 cells transfected with miR-217 or miR-NC after RIP. (D) The expression of miR-217 was examined in HK-2 cells transfected with pcDNA, ZEB1-AS1, si-NC or si- ZEB1-AS1 by qRT-PCR. * $P<0.05 ; * * P<0.01 ; * * * P<0.001$. 

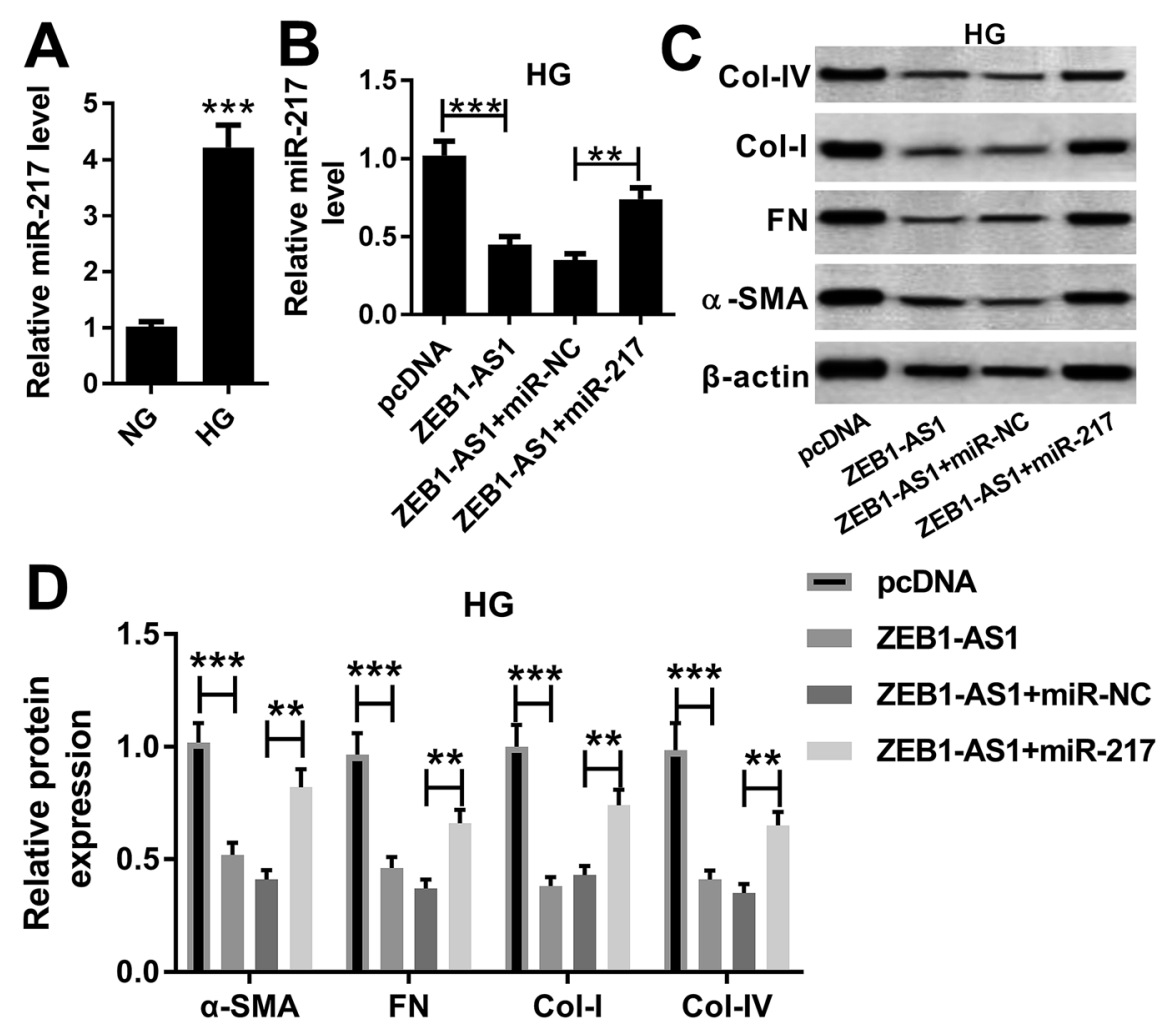

Fig. 5 Overexpressed ZEB1-AS1 protects against HG-induced renal fibrosis in HK-2 cells by regulating miR-217 inhibition. (A) The expression of miR-217 was determined using qRT-PCR in HK-2 cells treated with HG or NG. ZEB1-AS1, pCDNA, ZEB1-AS1 and miR-NC or ZEB1-AS1 and miR217 was transfected into HK-2 cells after treatment with HG. (B) The expression of miR-217 was detected using qRT-PCR. (C and D) The level of $\alpha$-SMA, fibronectin, collagen I and collagen IV was evaluated using the Western blot analysis. $* P<0.05 ; * * P<0.01 ; * * * P<0.001$.

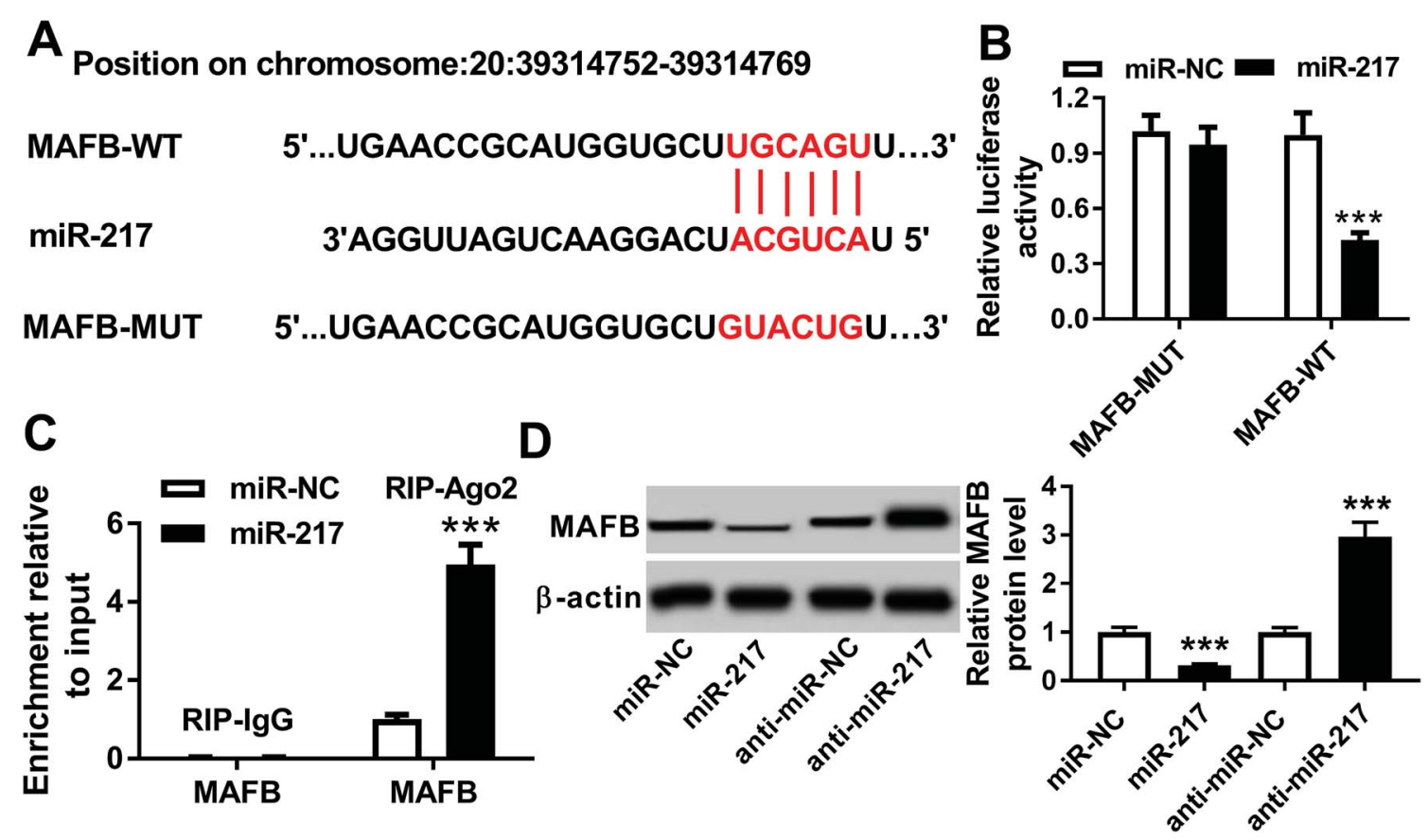

Fig. 6 MiR-217 directly targets MAFB and negatively regulates MAFB expression. (A) The putative binding sites of MAFB and miR-217 are listed. (B) Luciferase activity was investigated in HK-2 cells co-transfected with MAFB-WT or MAFB-MUT and miR-217 or miR-NC. (C) The enrichment of MAFB was analyzed in HK-2 cells transfected with miR-217 or miR-NC after RIP. (D) The expression of MAFB protein was examined in HK-2 cells transfected with miR-NC, miR-217, anti-miR-NC or anti-miR-217 by the Western blot analysis. $* P<0.05 ; * * P<0.01 ; * * * P<0.001$. 
markedly reduced the MAFB expression (Fig. 7A). To explore the interaction among ZEB1-AS1, miR-217 and MAFB in HG-induced renal fibrosis, anti-miR-217, anti-miR-NC, anti-miR-217 and si-NC, anti-miR-217 and si-MAFB, pcDNA, ZEB1-AS1, ZEB1-AS1 and siNC or ZEB1-AS1 and si-MAFB were transfected into HK-2 cells, respectively, after treating with $\mathrm{HG}$, and an elevated expression of MAFB protein induced by miR-217 inhibitor and ZEB1-AS1 was discovered (Fig. 7B and C) indicating that ZEB1-AS1 might regulate the MAFB expression by targeting miR-217. Moreover, we found that the $\alpha$-SMA, fibronectin, collagen I and IV protein expression was significantly inhibited by the miR-217 inhibitor, while the
MAFB deletion attenuated the miR-217 inhibitor-induced reduction of ECM proteins in HK-2 cells (Fig. 7D and E) suggesting that miR217 might promote HG-induced renal fibrosis in HK-2 cells via targeting the MAFB expression. Additionally, the MAFB deletion antagonized the effect of the overexpressed ZEB1-AS1-mediated protection against HG-induced renal fibrosis in HK-2 cells (Fig. 7F and G), illustrating that ZEB1-AS1 could exert its protective effects by modulating the MAFB expression. In summary, we verified the correlation between ZEB1-AS1, miR-217 and MAFB and identified that the overexpressed ZEB1-AS1 might protect against HG-induced renal fibrosis in HK-2 cells by regulating the miR-217/MAFB axis.
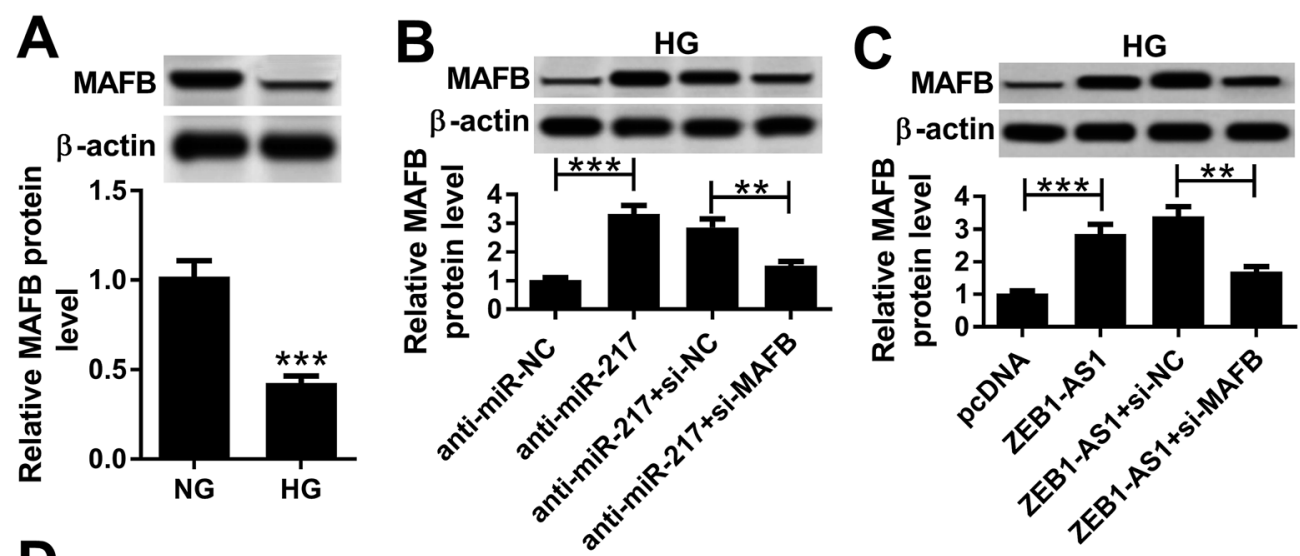

D

HG

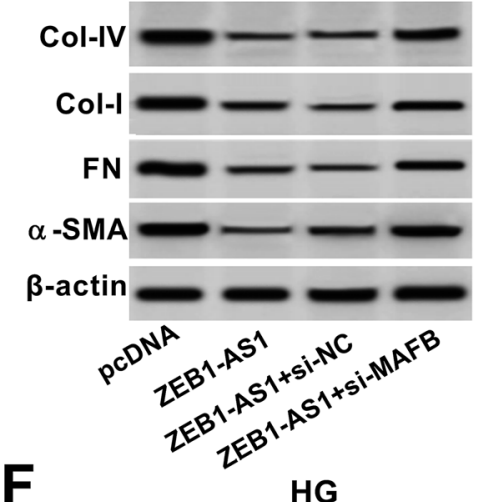

-

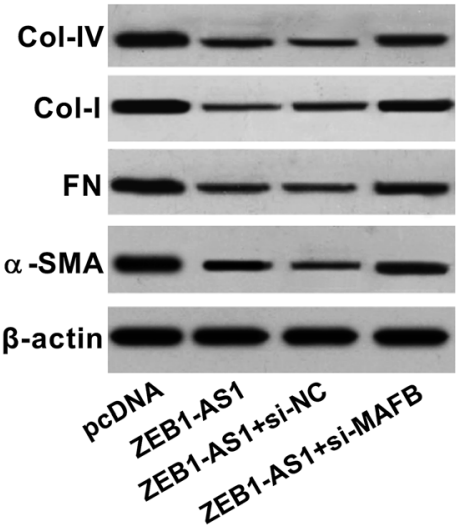

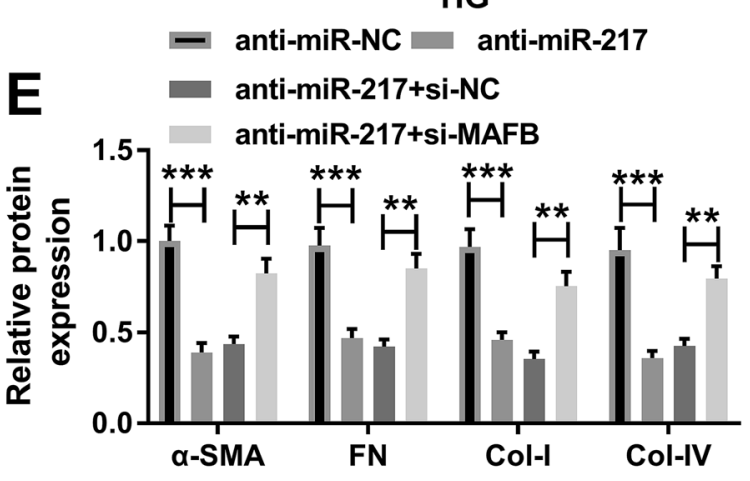

HG

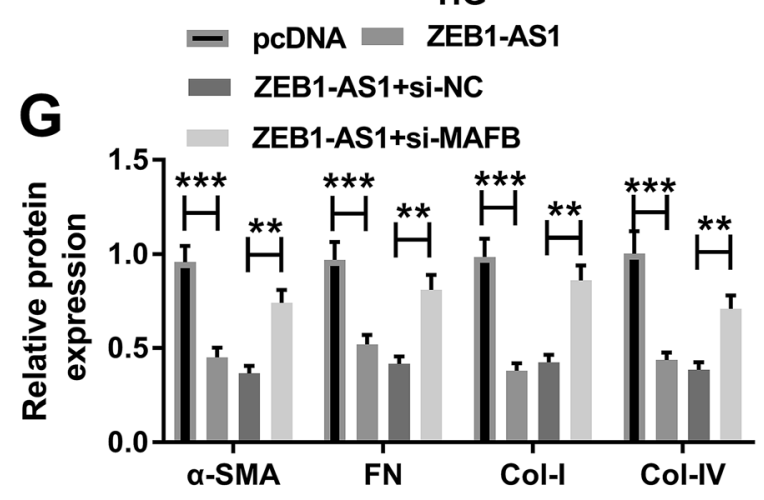

Fig. 7 Overexpressed ZEB1-AS1 protects against HG-induced renal fibrosis in HK-2 cells by regulating miR-217/MAFB axis. (A) The level of MAFB was determined using the Western blot analysis in HK-2 cells treated with HG or NG. Anti-miR-217, anti-miR-NC, anti-miR-217 and si-NC or anti-miR-217 and si-MAFB were transfected into HK-2 cells, respectively, after the treatment with HG. (B, D and E) The level of MAFB, $\alpha$-SMA, fibronectin, collagen I and collagen IV protein was detected using the Western blot analysis. The pcDNA, ZEB1-AS1, ZEB1-AS1 and si-NC or ZEB1AS1 and si-MAFB sequences were transfected into HK-2 cells, respectively, after the treatment with HG. (C, F and G) The level of MAFB, $\alpha$-SMA, fibronectin, collagen I and collagen IV protein was detected using the Western blot. $* P<0.05 ; * * P<0.01 ; * * P<0.001$. 


\section{Discussion}

Diabetic nephropathy is characterized by the accumulation of ECM proteins, such as fibronectin, collagen I and IV, and mesangial expansion in the kidney glomerular as well as tubular compartments, contributing to the end-stage renal disease progression. LncRNAs, one of the important components of noncoding RNA, bind to the target mRNAs and are considered to be critical gene regulators. ${ }^{24}$ LncRNAs are involved in various biological processes, such as gene expression and transcription, cell-cycle control, differentiation, epigenetic regulation, and immune response. ${ }^{25}$ Recently, an increasing number of studies have been performed to investigate the relationship between lncRNAs and DN. For example, lncRNA MALAT1 was dysregulated in DN and involved in high glucose-induced podocyte injury via its interplay with $\beta$-catenin. MALAT1 also antagonized renal tubular epithelial pyroptosis by modulated miR-23c targeting of ELAVL1 in DN. ${ }^{26,27}$ LncRNA CYP4B1-PS1-001 was significantly down-regulated in response to early DN in vitro and in vivo, while the overexpression of CYP4B1PS1-001 inhibited the proliferation and fibrosis of mesangial cells. $^{28}$ Li et al. proved that IncRNA LINC00968 accelerated the proliferation and fibrosis of mesangial cells by epigenetically repressing p21 via recruiting EZH2. ${ }^{29}$ Additionally, lncRNA TUG1 alleviated the ECM protein accumulation by mesangial cells, including PAI-1, TGF- $\beta 1$, fibronectin (FN) and collagen IV (Col IV), which was induced by the high glucose level in DN. ${ }^{30}$ Thus, lncRNA may be a novel target of DN pathogenesis.

Recently, the emerging studies identified that ZEB1-AS1 may contribute to the initiation or progression of cancers by positively modulating the expression of ZEB1. ${ }^{31,32}$ Furthermore, ZEB1 was reported to inhibit the TGF- $\beta 1$ mediated renal fibrosis, ${ }^{33}$ indicating the potential regulatory role of ZEB1-AS1 in renal fibrosis in DN. In the present study, we demonstrated that ZEB1-AS1 expression was down-regulated in the tissues of $\mathrm{DN}$ patients and $\mathrm{db} / \mathrm{db}$ diabetic mice as well as in HG-induced fibrosis in HK-2 cells. Subsequently, functional experiments indicated that the $\mathrm{db} / \mathrm{db}$ diabetic mice displayed higher levels of blood glucose, serum creatinine, and urinary albumin-to-creatinine ratio (ACR), which was obviously reversed by the ZEB1-AS1 treatment. Furthermore, the inhibition of the ECM protein expression, including $\alpha$-SMA, fibronectin, collagen I and IV, induced by the ZEB1-AS1 treatment, was detected in vivo and in vitro, indicating that ZEB1-AS1 could mitigate renal dysfunction and inhibit renal fibrosis in DN. Fibrosis is a hallmark of many cancers and is involved in the progression of cancers; therefore, it is a promising target in cancer treatment. ${ }^{34}$ Based on the previous and our results, ZEB1-AS1 was observed to be anti-fibrotic in DN. However, ZEB1-AS1 has been demonstrated to act as an oncogene in the progression of many types of tumors. ${ }^{8}$ The divergence of results may be explained by the following possible reasons. ${ }^{1}$ Even if ZEB1AS1 exhibits an anti-fibrotic effect on cancer, ZEB1-AS1 can also promote cancer development by regulating cell proliferation, migration, invasion or epithelial to mesenchymal transition (EMT) via interacting with targeted miRNAs or downstream signaling pathway. ${ }^{2}$ The function of ZEB1-AS1 may depend on the disease type, cellular context and the disease microenvironment because $\mathrm{DN}$ in patients is often accompanied by hyperglycemia, hypertension or dyslipidemia, which may affect its function. Nevertheless, the underlying mechanism of the ZEB1-AS1 function variability is complicated and needs further exploration.

LncRNAs are proposed to act as hosts for miRNAs. LncRNAs serve as modular molecules with individual domains that enable them to specifically associate with DNA, RNA and/or protein. ${ }^{35}$ In the current study, miR-217 was identified to be a putative target of ZEB1-AS1 via bioinformatics analysis. The level of miR-217 was discovered to be elevated in DN, and miR-217 functioned as a promoter of fibrosis in the high glucose induced mesangial cells. ${ }^{15,36}$ In addition, Han et al. found that miR-217 exhibited a protective effect on the dopamine D2 receptor in fibrosis in human renal proximal tubule cells by targeting TGF- $\beta$ or its downstream pathways. ${ }^{37}$ These findings indicated that miR-217 might be associated with the renal tubule cell fibrosis. In this study, we found that the level of miR-217 was significantly increased in the HG-induced HK-2 cells, indicating that miR-217 might function as an important regulator in the HG-induced tubule cell fibrosis. Subsequently, functional experiments showed that miR217 promoted the HG-induced renal fibrosis in HK-2 cells by promoting the ECM protein expression. Additionally, a correlation between ZEB1-AS1 and miR-217 was confirmed, and ZEB1-AS1 directly targeted the miR-217 and suppressed the miR-217 expression. Furthermore, rescue experiments indicated that ZEB1-AS1 induced the inhibition of the ECM protein expression by targeting the miR-217 expression in HK-2 cells.

MicroRNAs always bind to the $3^{\prime}$ UTR section of target genes and regulate the expression by repressing translation and/or inducing deadenylation as well as subsequent mRNA degradation. ${ }^{38}$ In this study, using bioinformatics analysis, we found that MAFB was a target of miR-217. MAFB was proposed to be associated with the development of DN in podocyte cells. Thus, we hypothesized that MAFB might be involved in renal tubule cell fibrosis of DN. Subsequently, the MAFB expression was found to be decreased in the HG-induced HK-2 cells and negatively regulated by miR-217. In addition, a rescue assay showed that MAFB deletion attenuated the miR-217 inhibitor-induced reduction of ECM proteins in HK-2 cells, suggesting that miR-217 might promote the HG-induced renal tubule cell fibrosis via targeting the MAFB expression. Moreover, an elevated expression of the MAFB protein, induced by the miR-217 inhibitor and ZEB1-AS1, was discovered, indicating the formation of ZEB1-AS1/miR-217/MAFB axis in the regulation of renal tubule cell fibrosis of DN. In addition, the gain- and loss-of function analysis illustrated that ZEB1-AS1 could exert its protective effect on the HG-induced HK-2 cells through modulating the MAFB expression.

To sum up, we illustrated that ZEB1-AS1could inhibit renal fibrosis of DN in vivo and in vitro. Furthermore, we demonstrated for the first time that overexpressed ZEB1-AS1 can protect against HGinduced fibrosis in renal proximal tubule cells by regulating the miR-217/MAFB axis. These findings might provide novel insight into the mechanism underlying HG-induced renal fibrosis in DN and contribute to the development of treatment approaches for DN.

\section{Disclosure statement}

The authors have nothing to disclose. 


\section{Conflict of interest}

There is no conflict of interest regarding the publication of this paper.

\section{References}

1 C. Magee, D. J. Grieve, C. J. Watson and D. P. Brazil, Cardiovasc. Drugs Ther., 2017, 31, 579-592.

2 C. Hu, L. Sun, L. Xiao, Y. Han, X. Fu, X. Xiong, X. Xu, Y. Liu, S. Yang, F. Liu and Y. S. Kanwar, Curr. Med. Chem., 2015, 22, 2858-2870.

3 I. Loeffler and G. Wolf, Cells, 2015, 4, 631-652.

4 Z. Lu, N. Liu and F. Wang, J. Diabetes Res., 2017, 2017, 7805058.

5 I. Ulitsky and D. P. Bartel, Cell, 2013, 154, 26-46.

6 J. Long and F. R. Danesh, Diabetes, 2018, 67, 552-553.

7 M. L. Alvarez and J. K. Distefano, Diabetes Res. Clin. Pract., 2013, 99, 1-11.

8 J. Li, Z. Li, K. Leng, Y. Xu, D. Ji, L. Huang, Y. Cui and X. Jiang, Cell Proliferation, 2018, 51, e12423.

9 C. Chen, Y. Feng and X. Wang, Clin. Chim. Acta, 2018, 484, 265-271.

10 J. Wang, J. Pang, H. Li, J. Long, F. Fang, J. Chen, X. Zhu, X. Xiang and D. Zhang, Mol. Ther. Nucleic Acids, 2018, 12, 741-750.

11 G. C. Shukla, J. Singh and S. Barik, Mol. Cell. Pharmacol., 2011, 3, 83-92.

12 H. Wu, L. Kong, S. Zhou, W. Cui, F. Xu, M. Luo, X. Li, Y. Tan and L. Miao, J. Diabetes Res., 2014, 2014, 920134.

13 Y. Shao, H. Ren, C. Lv, X. Ma, C. Wu and Q. Wang, Endocrine, 2017, 55, 130-138.

14 J. Sun, Z. P. Li, R. Q. Zhang and H. M. Zhang, Biochem. Biophys. Res. Commun., 2017, 483, 318-324.

15 Y. Shao, C. Lv, C. Wu, Y. Zhou and Q. Wang, Diabetes/Metab. Res. Rev., 2016, 32, 534-543.

16 K. Kataoka, M. Noda and M. Nishizawa, Mol. Cell. Biol., 1994, 14, 700-712.

17 T. Moriguchi, M. Hamada, N. Morito, T. Terunuma, K. Hasegawa, C. Zhang, T. Yokomizo, R. Esaki, E. Kuroda, K. Yoh, T. Kudo, M. Nagata, D. R. Greaves, J. D. Engel, M. Yamamoto and S. Takahashi, Mol. Cell. Biol., 2006, 26, 5715-5727.

18 Y. Sato, H. Tsukaguchi, H. Morita, K. Higasa, M. T. N. Tran, M. Hamada, T. Usui, N. Morito, S. Horita, T. Hayashi, J. Takagi, I. Yamaguchi, H. T. Nguyen, M. Harada, K. Inui, Y. Maruta, Y. Inoue, F. Koiwa, H. Sato, F. Matsuda, S. Ayabe, S. Mizuno, F. Sugiyama, S. Takahashi and A. Yoshimura, Kidney Int., 2018, 94, 396-407.

19 A. Zankl, E. L. Duncan, P. J. Leo, G. R. Clark, E. A. Glazov, M. C. Addor, T. Herlin, C. A. Kim, B. P. Leheup, J. McGill, S. McTaggart, S. Mittas, A. L. Mitchell, G. R. Mortier, S. P. Robertson, M. Schroeder, P. Terhal and M. A. Brown, Am. J. Hum. Genet., 2012, 90, 494-501.

20 N. Morito, K. Yoh, M. Ojima, M. Okamura, M. Nakamura, M. Hamada, H. Shimohata, T. Moriguchi, K. Yamagata and S. Takahashi, J. Am. Soc. Nephrol., 2014, 25, 2546-2557.
21 M. He, J. Wang, Z. Yin, Y. Zhao, H. Hou, J. Fan, H. Li, Z. Wen, J. Tang, Y. Wang, D. W. Wang and C. Chen, Aging, 2019, 11, 3055-3079.

22 Y. Li, D. Ren and G. Xu, IUBMB Life, 2019, 71, 873-881.

23 M. Zhan, I. M. Usman, L. Sun and Y. S. Kanwar, J. Am. Soc. Nephrol., 2015, 26, 1304-1321.

24 J. Whitehead, G. K. Pandey and C. Kanduri, Biochim. Biophys. Acta, 2009, 1790, 936-947.

25 O. Wapinski and H. Y. Chang, Trends Cell Biol., 2011, 21, 354-361.

26 X. Li, L. Zeng, C. Cao, C. Lu, W. Lian, J. Han, X. Zhang, J. Zhang, T. Tang and M. Li, Exp. Cell Res., 2017, 350, 327335.

27 M. Hu, R. Wang, X. Li, M. Fan, J. Lin, J. Zhen, L. Chen and Z. Lv, J. Cell Mol. Med., 2017, 21, 2732-2747.

28 M. Wang, S. Wang, D. Yao, Q. Yan and W. Lu, Mol. Cell. Endocrinol., 2016, 426, 136-145.

29 Z. Li, Z. Yu, X. Meng and P. Yu, Biochem. Biophys. Res. Commun., 2018, 504, 499-504.

30 L. J. Duan, M. Ding, L. J. Hou, Y. T. Cui, C. J. Li and D. M. Yu, Biochem. Biophys. Res. Commun., 2017, 484, 598-604.

31 W. Su, M. Xu, X. Chen, N. Chen, J. Gong, L. Nie, L. Li, X. Li, M. Zhang and Q. Zhou, Long noncoding RNA ZEB1-AS1 epigenetically regulates the expressions of ZEB1 and downstream molecules in prostate cancer, Mol. Cancer, 2017, 16(1), 142.

32 R. Qu, X. Chen and C. Zhang, LncRNA ZEB1-AS1/miR-4093p/ZEB1 feedback loop is involved in the progression of non-small cell lung cancer, Biochem. Biophys. Res. Commun., 2018, 507(1-4), 450-456.

33 S. Putta, L. Lanting, G. Sun, G. Lawson, M. Kato and R. Natarajan, Inhibiting microRNA-192 ameliorates renal fibrosis in diabetic nephropathy, J. Am. Soc. Nephrol., 2012, 23(3), 458-469.

34 M. Polasek, Y. Yang, D. T. Schuhle, M. A. Yaseen, Y. R. Kim, Y. S. Sung, A. R. Guimaraes and P. Caravan, Molecular MR imaging of fibrosis in a mouse model of pancreatic cancer, Sci. Rep., 2017, 7(1), 8114.

35 M. Kato and R. Natarajan, Diabetic nephropathy-emerging epigenetic mechanisms, Nat. Rev. Nephrol., 2014, 10(9), 517-530.

36 J. Sun, Z. P. Li, R. Q. Zhang and H. M. Zhang, Repression of miR-217 protects against high glucose-induced podocyte injury and insulin resistance by restoring PTEN-mediated autophagy pathway, Biochem. Biophys. Res. Commun., 2017, 483(1), 318-324.

37 F. Han, P. Konkalmatt, J. Chen, J. Gildea, R. A. Felder, P. A. Jose and I. Armando, MiR-217 mediates the protective effects of the dopamine D2 receptor on fibrosis in human renal proximal tubule cells, Hypertension, 2015, 65, 11181125.

38 M. Chekulaeva and W. Filipowicz, Mechanisms of miRNAmediated post-transcriptional regulation in animal cells, Curr. Opin. Cell Biol., 2009, 21(3), 452-460. 